\title{
Serum Phospholipid Transfer Protein Mass as a Possible Protective Factor for Coronary Heart Diseases
}

\author{
Hiroshi Yatsuya, MD; Koji Tamakoshi, MD; Hiroaki Hattori, PhD*; Rei Otsuka, BSc; \\ Keiko Wada, MD; Huiming Zhang, MD; Tomoko Mabuchi, BSc; \\ Miyuki Ishikawa, ME; Chiyoe Murata, MPH; Tsutomu Yoshida, MD**; \\ Takaaki Kondo, MD; Hideaki Toyoshima, MD
}

\begin{abstract}
Background Phospholipid transfer protein (PLTP) can generate pre $\beta$ high-density lipoprotein (HDL), an efficient acceptor of peripheral cholesterol, by mediating a process called HDL conversion. The transfer of phospholipids to immature HDL is also essential in maintaining reverse cholesterol transport. The phospholipid transfer activity of PLTP has been associated with various patho-physiological conditions; however, little information is available concerning the relationship between PLTP mass and disease.

Methods and Results Using a sandwich enzyme-linked immunosorbent assay, PLTP concentration was measured and related to the risk of developing cardiovascular disease in a worksite-based cohort of Japanese men $(n=2,567)$. Multiple linear regression analysis showed significant associations between PLTP and HDL cholesterol, triglycerides, low-density lipoprotein cholesterol, and body mass index (standardized $\beta=0.395$, $-0.191,-0.064$, and -0.064 , respectively; $\mathrm{R}^{2}=0.31$ ). During the follow-up period, there were 10 cases of coronary heart disease (CHD) and 7 of stroke. The multivariate adjusted relative risk of CHD was 0.46 (95\% confidence interval, $0.20-1.07)$ for an increase of 1 standard deviation in the PLTP value ( $\mathrm{p}=0.071)$. PLTP concentration was not related to the risk of stroke.

Conclusions The results of this prospective study indicate that the serum PLTP concentration would serve as a predictor of CHD, independent of HDL cholesterol, triglycerides and other established risk factors. (Circ J 2004; 68: 11-16)
\end{abstract}

Key Words: Cohort study; Coronary heart disease; High-density lipoprotein; Japan; Phospholipid transfer protein

$A^{n}$

inverse relationship between high-density lipoprotein (HDL) cholesterol and cardiovascular diseases has been demonstrated in epidemiologic studies, 1,2 and is often explained by the capacity of HDL to transport cholesterol from peripheral tissues to the liver, known as reverse cholesterol transport? In this process, pre $\beta$-HDL, a quantitatively minor subfraction of HDL, is thought to play a critical role because it acts as an efficient acceptor in the cholesterol efflux process from the plasma membrane of peripheral cells?,4 Phospholipid transfer protein (PLTP) is capable of generating pre $\beta$-HDL by inducing HDL conversion, a process that remodels a homogeneous HDL fraction into populations of large and small HDL particles5-8 The transfer of phospholipids to immature HDL in the process of HDL maturation is also essential in maintaining reverse cholesterol transport5,9,10 In addition, PLTP has other roles, such as the transfer of atocopherol to cell membrane for the maintenance of endothelial function, or the transfer of lipopolysaccharide to

(Received July 17, 2003; revised manuscript received September 30, 2003; accepted October 10, 2003)

Department of Public Health/Health Information Dynamics, Nagoya University Graduate School of Medicine, Nagoya, *Research Division, R\&D Center, BML, Inc, Kawagoe and **Department of Public Health, Fujita Health University, Toyoake, Japan

Mailing address: Hideaki Toyoshima, MD, PhD, Department of Public Health, Nagoya University Graduate School of Medicine, 65 Tsurumai-cho, Showa-ku, Nagoya 466-8550, Japan. E-mail: h828@ med.nagoya-u.ac.jp lipoproteins, which leads to neutralization of its potent inflammatory function? Altogether, PLTP can be considered to play an important role in the prevention of atherosclerosis, and thus it has recently attracted much attention.

Previous studies regarding PLTP examined its phospholipid transfer activity in patho-physiologic states in human or experimental models! ${ }^{1-14}$ Sandwich enzyme-linked immunosorbent assay with 2 monoclonal antibodies to human PLTP has been recently introduced to assay PLTP concentration, 15,16 but the significance of the PLTP concentration in human diseases is incompletely understood $5,17 \mathrm{It}$ is, therefore, important to evaluate whether an elevated concentration of serum PLTP is associated with an increased or decreased risk of cardiovascular diseases. We examined serum PLTP in relation to newly diagnosed cardiovascular diseases in a middle-aged worksite-based cohort of men in Japan. We also examined the association of serum PLTP concentration with other cardiovascular risk factors.

\section{Methods}

\section{Subjects}

The present analysis is based on a cohort of male workers in a worksite in Aichi prefecture, central Japan!18,19 Its aim was to determine factors associated with the development of cardiovascular diseases. The study began in 1997, when 2,896 male employees of a manufacturing company, aged 34-59 years, were recruited as potential participants. A self-administered questionnaire was used to assess the 
Table 1 Baseline Characteristics of the Study Subjects

\begin{tabular}{|c|c|c|c|c|}
\hline & \multirow{2}{*}{$\begin{array}{c}\text { Total } \\
(n=2,567)\end{array}$} & \multicolumn{3}{|c|}{ Cases } \\
\hline & & $\begin{array}{c}\text { Any cardiovascular diseases } \\
(n=17)\end{array}$ & $\begin{array}{c}\text { CHD } \\
(n=10)\end{array}$ & $\begin{array}{l}\text { Stroke } \\
(n=7)\end{array}$ \\
\hline Age (years) & $50 \pm 7$ & $52 \pm 5$ & $52 \pm 6$ & $52 \pm 3$ \\
\hline$P L T P(\mu \mathrm{g} / \mathrm{ml})$ & $14.9 \pm 4.3$ & $13.1 \pm 3.8$ & $12.2 \pm 2.5$ & $14.4 \pm 5.1$ \\
\hline Body mass index $\left(\mathrm{kg} / \mathrm{m}^{2}\right)$ & $22.8 \pm 2.7$ & $23.4 \pm 3.7$ & $23.5 \pm 4.3$ & $23.4 \pm 3.0$ \\
\hline Systolic blood pressure $(\mathrm{mmHg})$ & $129 \pm 19$ & $143 \pm 15$ & $141 \pm 13$ & $145 \pm 19$ \\
\hline Glycosylated hemoglobin (\%) & $5.7 \pm 0.6$ & $6.5 \pm 1.3$ & $6.6 \pm 1.5$ & $6.3 \pm 1.1$ \\
\hline HDL cholesterol $(\mathrm{mg} / \mathrm{dl})$ & $54 \pm 14$ & $46 \pm 11$ & $46 \pm 12$ & $46 \pm 11$ \\
\hline LDL cholesterol $(\mathrm{mg} / \mathrm{dl})$ & $128 \pm 31$ & $136 \pm 30$ & $150 \pm 20$ & $117 \pm 31$ \\
\hline Triglycerides $(\mathrm{mg} / \mathrm{dl})$ & $132 \pm 94$ & $114 \pm 41$ & $130 \pm 60$ & $194 \pm 131$ \\
\hline \multicolumn{5}{|l|}{ Smoking status (\%) } \\
\hline Never & 33.6 & 31.3 & 30.0 & 33.3 \\
\hline Past smoker & 13.2 & 6.3 & 10.0 & 0.0 \\
\hline \multicolumn{5}{|l|}{ Current smoker } \\
\hline 1-24 cigarettes/day & 36.0 & 31.3 & 20.0 & 50.0 \\
\hline$\geq 25$ cigarettes $/$ day & 17.2 & 31.3 & 40.0 & 16.7 \\
\hline \multicolumn{5}{|l|}{ Alcohol intake (\%) } \\
\hline None & 26.4 & 23.5 & 20.0 & 28.6 \\
\hline Light & 21.8 & 23.5 & 30.0 & 14.3 \\
\hline Moderatte & 26.0 & 35.3 & 40.0 & 28.6 \\
\hline Heavy & 25.8 & 17.6 & 10.0 & 28.6 \\
\hline \multicolumn{5}{|l|}{ Leisure-time physical activity (\%) } \\
\hline Not very active & 82.8 & 76.5 & 80.0 & 71.4 \\
\hline Somewhat active & 12.6 & 5.9 & 0.0 & 14.3 \\
\hline Regularly active & 4.5 & 17.6 & 20.0 & 14.3 \\
\hline
\end{tabular}

PLTP, phospholipid transfer protein; CHD, coronary heart diseases; HDL, high-density lipoprotein; LDL, low-density lipoprotein. Data are expressed as means \pm standard deviations.

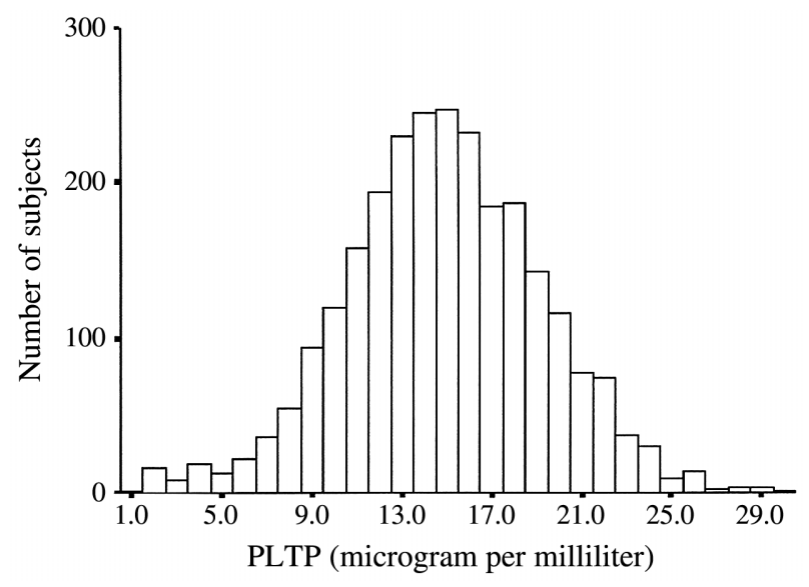

Fig 1. Distribution of phospholipid transfer protein (PLTP).

baseline characteristics of the participants. Of the 2,896 workers, $2,567(88.6 \%)$ participated in the study by completing a questionnaire and donating a residual serum sample, which was taken during an annual health check-up after fasting at least $12 \mathrm{~h}$ overnight. Samples were kept frozen in a $-80^{\circ} \mathrm{C}$ deep freezer immediately after centrifuging until determination of serum PLTP concentration in the year 2000. The study protocol and informed consent procedure was approved by the ethics committee of Nagoya University Graduate School of Medicine.

\section{Follow up and Identification of Cases of Cardiovascular Diseases}

All participants were followed from the date of enrollment until the date of the last health check-up unless they had developed one of the prospectively defined endpoints, which were reported by the healthcare professionals of the worksite using a standardized form. The median follow-up period was 37 months. Cardiovascular diseases here refer to coronary heart diseases (CHD), identified as myocardial infarction or cardiac sudden death, and stroke. Medical and surgical interventions, such as percutaneous transcatheter angioplasty and coronary artery bypass graft, were also regarded as CHD events. Myocardial infarction included definite fatal and non-fatal myocardial infarction according to the MONICA criteria. ${ }^{20}$ Stroke included cerebral infarction, cerebral hemorrhage, and subarachnoid hemorrhage. Sudden cardiac death was defined as sudden unexpected death within $1 \mathrm{~h}$ of the onset of acute symptoms and signs. The diagnosis was confirmed by a medical or death certificate.

\section{Serum PLTP Concentration and Phospholipid Transfer Activity of PLTP}

Serum PLTP concentration was measured by a sandwich enzyme-linked immunosorbent assay method using 2 monoclonal antibodies specific to PLTP ${ }^{15}$ Circulating PLTP has been demonstrated to be present in 2 forms, one catalytically active and the other inactive 11,22 The assay measures the total concentration of PLTP. The assay range was $1.2-30.0 \mathrm{mg} / \mathrm{L}$, and the intra- and inter-assay coefficients of variations were $<3.0 \%$ and $<4.2 \%$, respectively.

Phospholipid transfer activity of PLTP was measured by a liposome-HDL3 system as previously described 15 The activity was expressed as $\mu$ mol of phosphatidylcholine transferred to HDL3 per milliliter of plasma per hour. The activity was measured only in a sample of 41 subjects, which consisted of 32 normolipidemics and 9 dyslipidemics (triglyceride concentration $\geq 150 \mathrm{mg} / \mathrm{dl}$ and HDL cholester$\mathrm{ol}<40 \mathrm{mg} / \mathrm{dl}$ ) for the purpose of describing the correlation with circulating PLTP concentration. 
Table 2 Correlation Coefficients Between the Serum PLTP Concentration and Age, Body Mass Index, and Other Cardiovascular Risk Factors

\begin{tabular}{lcccccc}
\hline \hline Age & BMI & SBP & HbAlc & LDLC & HDLC & $T G$ \\
\hline-0.03 & $-0.27^{*}$ & $-0.09^{*}$ & $-0.11^{*}$ & $-0.18^{*}$ & $0.51^{*}$ & $-0.41^{*}$ \\
\hline${ }^{*} p<0.001$. & \\
PLTP, phospholipid transfer protein; & BMI, body mass index; & SBP, systolic blood pressure; HbAlc, glycosilated hemoglobin; LDLC, \\
low-density lipoprotein cholesterol; HDLC, high-density lipoprotein cholesterol; TG, triglycerides.
\end{tabular}

Table 3 Parameter Estimates of Stepwise Multiple Regression With Serum PLTP as the Dependent Variable

\begin{tabular}{lcl}
\hline \hline Variable & Standardized $\beta$ & $p$ value \\
\hline HDLC & 0.395 & $<0.0001$ \\
$T G$ & -0.191 & $<0.0001$ \\
LDLC & -0.064 & $<0.001$ \\
BMI & -0.064 & $<0.001$ \\
\hline
\end{tabular}

$R^{2}=0.31$.

Independent variables excluded from the model were systolic blood pressure, glycosilated hemoglobin, and age.

PLTP, phospholipid transfer protein; HDLC, high-density lipoprotein cholesterol; TG, triglycerides; LDLC, low-density lipoprotein cholesterol; BMI, body mass index.

\section{Definition of Covariates}

The study participants provided information on their smoking history, drinking habits, and physical activity. Smoking status was classified into 4 levels (never, past, and current smokers of $1-24$ cigarettes or $\geq 25$ per day). Drinking habit was first assessed by the number of drinking days per week (none, 1-3, 4-6, and daily) ${ }^{23}$ If present (not none), it was further categorized into 3 levels by weekly consumption (light, moderate, heavy); that is, daily alcohol consumption multiplied by days of drinking per week. Leisure-time physical activity was assessed by 2 questions: frequency (seldom, 1-3 times per month, 1-2 times per week, $\geq 3$ times per week) and intensity (vigorous, moderate, light). Those who engaged in vigorous activity $1-2$ times or more per week, or moderate activity 3 times or more per week, were classified as 'regularly active'. Those who engaged in vigorous activity 1-3 times per month, moderate activity 1-2 times per week, or light activity 3 times or more per week were classified as 'somewhat active'. All others with no missing data on these questions were classified as 'not very active'. Vigorous activity was defined in the questionnaire as the level that leaves participants out of breath. Similarly, moderate activity was defined as the level that leaves participants breathing rather hard.

Body-mass index (BMI) was calculated as weight in kilograms divided by the square of the height in meters. Serum level of low-density lipoprotein cholesterol (LDLC), HDL cholesterol (HDL-C) and triglycerides (TG) were measured enzymatically. Glycosylated hemoglobin concentration was analyzed by high-performance liquid chromatography. Serum TG concentration was natural logarithmically transformed to approximately normalize the distribution.

\section{Statistical Analysis}

Forward stepwise multiple regression with serum PLTP concentration as the dependent variable was used in order to evaluate associations of PLTP with established cardiovascular risk factors. Independent variables entered into the model included age, systolic blood pressure, BMI, LDL-C, HDL-C, TG and glycosylated hemoglobin. A selection threshold of a p-value of less than 0.05 was employed.
Cox proportional-hazards regression models were used to calculate the relative risks (RR) for CHD, stroke and cardiovascular diseases in relation to serum PLTP concentration. Because the distribution of serum PLTP was normally distributed, we treated it as a continuous variable, and presented the RR that represent change in risk per increment of $1 \mathrm{SD}$ (4.3), which was calculated from the distribution in all subjects. Adjusted estimates of risk were obtained with multivariate models that also controlled for age, smoking status, alcohol intake, and leisure-time physical activity (multivariate model 1), variables entered in the multivariate model 1, BMI, systolic blood pressure, LDL-C, and glycosylated hemoglobin (multivariate model 2), and variables entered in the multivariate model 2, HDL-C and TG (multivariate model 3 ).

All reported p-values were 2-sided and a p-value of less than 0.05 was considered statistically significant. However, a p-value of less than 0.1 was treated as of marginal significance. The 95 percent confidence intervals $(95 \% \mathrm{CI})$ are presented for all RR. All statistical analyses were performed with the SPSS statistical package for Windows, version 11.5 (Chicago, IL, USA).

\section{Results}

The baseline characteristics of the participants are presented in Table 1 . The mean serum PLTP concentration was $14.9 \mu \mathrm{g} / \mathrm{ml}$. The PLTP was normally distributed (Kolmogorov-Smirnov test; range 1.2-29.8) (Fig 1). There was no correlation between the serum PLTP concentration and age (Pearson's $r=-0.03$, NS) (Table 2). There was a strong positive correlation between the PLTP and serum HDL-C concentrations (Pearson's $\mathrm{r}=0.51, \mathrm{p}<0.001$ ). There was a significant negative correlation between the PLTP concentration and TG (Pearson's $r=-0.41, \mathrm{p}<0.001)$. Significant correlation was not found between PLTP concentration and PLTP activity in normolipidemics nor in dyslipidemics (Spearman's $\mathrm{r}=0.230, \mathrm{p}=0.21 \quad(\mathrm{n}=32)$ and $\mathrm{r}=-0.092, \mathrm{p}=0.81 \quad(\mathrm{n}=9)$, respectively). Stepwise multiple linear regression analysis performed with serum PLTP concentration as the dependent variable and with age, BMI, systolic blood pressure, LDL-C, HDL-C, TG and glycosylated hemoglobin as independent variables, showed signifi- 
Table 4 Cox Proportional Hazard Models Examining the Relation Between the Serum PLTP and the Risk of Cardiovascular Diseases*

\begin{tabular}{|c|c|c|c|c|c|c|c|c|c|}
\hline \multirow[b]{2}{*}{ Variables adjusted for } & \multicolumn{3}{|c|}{ Any cardiovascular diseases } & \multicolumn{3}{|c|}{ Coronary heart diseases } & \multicolumn{3}{|c|}{ Stroke } \\
\hline & $\begin{array}{l}\text { No. of Cases/ } \\
\text { No. of Subjects }\end{array}$ & $\begin{array}{c}R R \\
(95 \% C I)\end{array}$ & $p$ value & $\begin{array}{l}\text { No. of Cases/ } \\
\text { No. of Subjects }\end{array}$ & $\begin{array}{c}R R \\
(95 \% C I)\end{array}$ & $p$ value & $\begin{array}{l}\text { No. of Cases/ } \\
\text { No. of Subjects }\end{array}$ & $\begin{array}{c}R R \\
(95 \% C I)\end{array}$ & $p$ value \\
\hline Univariate & $17 / 2,567$ & $0.66(0.41-1.07)$ & 0.093 & $10 / 2,567$ & $0.52(0.28-0.97)$ & 0.039 & $7 / 2,567$ & $0.92(0.45-1.91)$ & 0.830 \\
\hline Multivariate model 1 & $16 / 2,494$ & $0.59(0.36-0.98)$ & 0.039 & $10 / 2,494$ & $0.49(0.25-0.93)$ & 0.029 & $6 / 2,494$ & $0.76(0.34-1.70)$ & 0.498 \\
\hline Multivariate model 2 & $15 / 2,493$ & $0.60(0.34-1.04)$ & 0.069 & $9 / 2,493$ & $0.45(0.21-0.97)$ & 0.041 & $6 / 2,493$ & $0.80(0.35-1.84)$ & 0.593 \\
\hline Multivariate model 3 & $15 / 2,493$ & $0.73(0.37-1.44)$ & 0.371 & $9 / 2,493$ & $0.46(0.20-1.07)$ & 0.071 & $6 / 2,493$ & $1.37(0.50-3.76)$ & 0.545 \\
\hline
\end{tabular}

*The concentration of phospholipid transfer protein was analyzed as a continuous variable.

The relative risks (RR) are per increment of 1 SD (4.3) in PLTP value. CI, confidence interval; PLTP, phospholipid transfer protein.

Multivariate model 1 adjusted for age, smoking status, alcohol intake, and leisure-time physical activity.

Multivariate model 2 adjusted for variables entered in the multivariate model 1, body mass index, systolic blood pressure, low-density lipoprotein cholesterol, and glycosylated hemoglobin.

Multivariate model 3 adjusted for variables entered in the multivariate model 2, HDL cholesterol and triglycerides.

cant and independent associations between serum PLTP concentration and HDL-C, TG, LDL-C, and BMI (standardized $\beta=0.395,-0.191,-0.064$, and -0.064 , respectively) (Table 3). Thirty-one percent of the variance in serum PLTP concentration was explained by this model; however, $26 \%$ of variance was explained only by HDL-C, and an additional $4 \%$ was explained by TG.

During follow-up of 7,037 person-years, CHD developed in 10 subjects ( 8 cases of non-fatal acute myocardial infarction, 1 of unstable angina, which ended up as coronary artery bypass graft operation, and 1 sudden cardiac death), and stroke developed in 7 subjects (5 cases of nonfatal cerebral infarction and 2 of fatal subarachnoid hemorrhage). Crude incidence rates for $\mathrm{CHD}$ and stroke were 142.1 (per 100,000 person-years) and 99.5, respectively and the age-adjusted incidence rates to the world standard population $^{24}$ were 112.9 (per 100,000 person-years) and 62.1 , respectively.

The results relating the serum PLTP to the development of any cardiovascular diseases, CHD or stroke are shown in Table 4. The concentration of serum PLTP was significantly and inversely related to the risk of cardiovascular diseases in the multivariate model $1(\mathrm{p}=0.039)$. In the multivariate model 2 adjusting for BMI, systolic blood pressure, LDL-C, and glycosylated hemoglobin, a marginally significant association was observed $(\mathrm{p}=0.069)$. Further adjustment with TG and HDL-C (multivariate model 3) substantially attenuated the association. The concentration of serum PLTP was, however, inversely related to the risk of CHD with marginal significance even after adjustment for HDL-C and TG. The relative risk of CHD in the multivariate model 3 was 0.46 (95\%CI $0.20-1.07)$ for an increase of 1 SD in the PLTP value $(\mathrm{p}=0.071)$. The concentration of serum PLTP was not related to the risk of stroke $(\mathrm{p}=0.545$ in multivariate model 3$)$.

\section{Discussion}

The results of this prospective, observational study indicate that there is a graded inverse association between serum PLTP concentration and the risk of cardiovascular disease, especially CHD. The observed association appeared to be independent of age and other covariates included in the multivariate models. In the analysis adjusted for the concentration of HDL-C and TG, which explained $30 \%$ of the variance in PLTP concentration, there was still a marginally significant association between serum PLTP concentration and the risk of CHD. The reason for the lack of association between stroke and the
PLTP concentration is unclear. Though the limited number of stroke cases makes it difficult to speculate specifically, classic risk factors such as systolic blood pressure or glycosylated hemoglobin had a significant, or marginally significant, direct association with the risk of stroke (relative risk of $1.04(\mathrm{p}=0.06)$ for the increment of $1 \mathrm{mmHg}$ in blood pressure, and $2.13(\mathrm{p}=0.02)$ for the increment of $1 \%$ in glycosylated hemoglobin, in the multivariate model 3 ). Exclusion of 2 cases of subarachnoid hemorrhage, which may have a different etiology from cerebral infarction, did not alter the association (data not shown). To elucidate whether the association of serum PLTP concentration exists only with the development of CHD, further investigations with more cases are needed.

This is the first report showing a significant inverse relationship between the serum concentration of PLTP mass and the development of CHD. Previous studies regarding PLTP and CHD or other patho-physiologic conditions have mainly focused on its phospholipid transfer activity 25,26 The assay used in the present study measures the total amount of serum PLTP mass, and it is reported that it does not differentiate the catalytically active form from the inactive form of PLTP22 It is also suggested that majority of the PLTP mass exists in the inactive form in the circulation in healthy subjects! ${ }^{16,17}$ We obtained the data regarding PLTP activity in a sample of subjects, and the results indicated that the concentration of serum PLTP mass did not correlate with its activity. Thus, the inverse association between PLTP mass and CHD found in this study may not be solely attributed to the phospholipid transfer activity of PLTP.

The reason for the presence of PLTP with low phospholipid transfer activity is not yet clear. Oka et al found that PLTP activity in hypoalphalipoproteinemia was comparable to that in normolipidemic controls, although PLTP concentration was significantly lower in hypoalphalipoproteinemia subjects than in controls 17 They found that the low PLTP concentration in hypoalphalipoproteinemia was because of a lack of inactive PLTP. Dyslipidemic states, such as low HDL-C or elevated TG, ${ }^{16,22}$ or intravenous infusion of apo A-I/phosphatidylcholine discs, which increases the plasma pre $\beta$-apo A-I and HDL-C concentrations, ${ }^{27}$ were demonstrated to modify the distribution of active and inactive pools of PLTP. It is, therefore, necessary to conduct further research to elucidate the mechanisms involved in the existence of inactive PLTP, with regard to its function in lipoprotein metabolism. Clinical studies that measure both the activity and the concentration of PLTP are also needed to understand the reason for the disparity between them in various pathophysiological 
settings. Nevertheless, the present results show that the serum PLTP concentration, which probably reflects the amount of the inactive form of PLTP, was significantly and inversely associated with the development of $\mathrm{CHD}$, even after adjustment of HDL-C and TG.

Our finding that an elevated concentration of PLTP may be a protective marker of CHD is consistent with several previous reports that indicated that the function of PLTP was anti-atherogenic, ${ }^{7,28}$ although all these studies did not obtain the concentration of the PLTP mass, except for a few ${ }^{27}$ which may limit direct comparisons. Haperen et al showed that accumulation of cholesterol in peritoneal macrophages was significantly reduced in transgenic mice that overexpressed human $\mathrm{PLTP}^{7}$ and that result was attributed to the increased production of pre $\beta$-HDL in the transgenic mice. In contrast, overexpression of PLTP in mice heterozygous for the LDL receptor, which is a model for atherosclerosis, significantly increased the area of the atherosclerotic lesion in the mice 26 However, these apparently inconsistent results were from experiments with specific mouse models, and may be attributable to differences in the experimental models?,29 Because studies regarding PLTP and atherosclerosis in humans are scarce, further research must evaluate the present result.

One mechanism that mediates the possible protective effect of PLTP on the risk of CHD is probably the capability of PLTP to generate pre $\beta$-HDL, an efficient acceptor of peripheral cell cholesterol in reverse cholesterol transport. The pre $\beta$-HDL concentration has been found to be significantly lower in CHD cases than in healthy controls 30 Plasma from the transgenic mouse that overexpressed human PLTP had increased potential for pre $\beta$-HDL formation?,31 Furthermore, generation of pre $\beta$-HDL, measured as the change in pre $\beta$-HDL concentration during incubation of plasma from cases and controls, which would reflect a function of PLTP, was significantly higher in controls than in cases. 30

The prospective nature of the present study between newly developed CHD and serum PLTP concentration suggests that the decrease in the PLTP concentration preceded the onset of CHD. However, we measured PLTP concentrations only at the time of enrollment and there is still uncertainty about how long this inter-individual variation persisted. Further studies to examine the degree of the intra-individual variability of the PLTP concentration over varying time intervals are needed.

Other limitations of the study are the relatively short follow-up period and that the study population included only Japanese males. Although the study is limited in the number of cases included, the incidence rates of CHD and stroke were both comparable to those of previous reports on Japanese males of approximately the same age range 32,33 However, the relation between elevated serum PLTP concentrations and cardiovascular diseases must be evaluated in other cohort studies. If such studies confirm our findings, proof of a causal association between serum PLTP and the development of cardiovascular diseases will require further elucidation of the patho-physiologic mechanisms. Furthermore, studies to investigate the association between lifestyle factors and the serum PLTP concentrations are needed for the development of practical prevention programs.

\section{Acknowledgments}

The authors express their sincere appreciation to Dr Noboru Okamoto, Dr Junji Toyama, and the late Dr Shogo Shima who contributed to the initiation of the present cohort study. We also thank the participants and the healthcare professionals of the company.

The work was funded by Grants 13770192, 13470087, and 12670352 from the Ministry of Education, Culture, Sports, Science and Technology of Japan, Japan Atherosclerosis Prevention Fund (JAPF), and an unrestricted grant from Banyu Pharmaceutical Co, Ltd.

\section{References}

1. Mabuchi H, Kita T, Matsuzaki M, Matsuzawa Y, Nakaya N, Oikawa $\mathrm{S}$, et al. Large scale cohort study of the relationship between serum cholesterol concentration and coronary events with low-dose simvastatin therapy in Japanese patients with hypercholesterolemia and coronary heart disease: Secondary prevention cohort study of the Japan Lipid Intervention Trial (J-LIT). Circ J 2002; 66: 1096-1100.

2. Sacks FM. The role of high-density lipoprotein (HDL) cholesterol in the prevention and treatment of coronary heart disease: Expert group recommendations. Am J Cardiol 2002; 90: 139-143.

3. Sviridov D, Nestel P. Dynamics of reverse cholesterol transport: Protection against atherosclerosis. Atherosclerosis 2002; 161: 245-254.

4. Nanjee MN, Cooke CJ, Garvin R, Semeria F, Lewis G, Olszewski $\mathrm{WL}$, et al. Intravenous apoA-I/lecithin discs increase pre-beta-HDL concentration in tissue fluid and stimulate reverse cholesterol transport in humans. J Lipid Res 2001; 42: 1586-1593.

5. Huuskonen J, Olkkonen VM, Jauhiainen M, Ehnholm C. The impact of phospholipid transfer protein (PLTP) on HDL metabolism. Atherosclerosis 2001; 155: 269-281.

6. Settasatian N, Duong M, Curtiss LK, Ehnholm C, Jauhiainen M, Huuskonen J, et al. The mechanism of the remodeling of high density lipoproteins by phospholipid transfer protein. J Biol Chem 2001; 276: $26898-26905$.

7. van Haperen R, van Tol A, Vermeulen P, Jauhiainen M, van Gent T, van den Berg P, et al. Human plasma phospholipid transfer protein increases the antiatherogenic potential of high density lipoproteins in transgenic mice. Arterioscler Thromb Vasc Biol 2000; 20: $1082-$ 1088 .

8. Lie J, de Crom R, Jauhiainen M, van Gent T, van Haperen R, Scheek $\mathrm{L}$, et al. Evaluation of phospholipid transfer protein and cholesteryl ester transfer protein as contributors to the generation of pre betahigh-density lipoproteins. Biochem J 2001; 360: 379-385.

9. van Tol A. Phospholipid transfer protein. Curr Opin Lipidol 2002; 13: $135-139$.

10. Fournier N, Atger V, Cogny A, Vedie B, Giral P, Simon A, et al. Analysis of the relationship between triglyceridemia and HDL-phospholipid concentrations: Consequences on the efflux capacity of serum in the Fu5AH system. Atherosclerosis 2001; 157: 315-323.

11. Beentjes JA, van Tol A, Sluiter WJ, Dullaart RP. Low plasma lecithin: Cholesterol acyltransferase and lipid transfer protein activities in growth hormone deficient and acromegalic men: Role in altered high density lipoproteins. Atherosclerosis 2000; 153: 491 498.

12. Colhoun HM, Scheek LM, Rubens MB, Van Gent T, Underwood $\mathrm{SR}$, Fuller JH, et al. Lipid transfer protein activities in type 1 diabetic patients without renal failure and nondiabetic control subjects and their association with coronary artery calcification. Diabetes 2001; 50: $652-659$.

13. Riemens SC, van Tol A, Sluiter WJ, Dullaart RP. Plasma phospholipid transfer protein activity is lowered by 24-h insulin and acipimox administration: Blunted response to insulin in type 2 diabetic patients. Diabetes 1999; 48: 1631-1637.

14. Dullaart RP, van Tol A. Twenty four hour insulin infusion impairs the ability of plasma from healthy subjects and Type 2 diabetic patients to promote cellular cholesterol efflux. Atherosclerosis 2001; 157: $49-56$.

15. Oka T, Kujiraoka T, Ito M, Nagano M, Ishihara M, Iwasaki T, et al. Measurement of human plasma phospholipid transfer protein by sandwich ELISA. Clin Chem 2000; 46: 1357-1364.

16. Huuskonen J, Ekstrom M, Tahvanainen E, Vainio A, Metso J, Pussinen P, et al. Quantification of human plasma phospholipid transfer protein (PLTP): Relationship between PLTP mass and phospholipid transfer activity. Atherosclerosis 2000; 151: 451-461.

17. Oka T, Yamashita S, Kujiraoka T, Ito M, Nagano M, Sagehashi Y, et al. Distribution of human plasma PLTP mass and activity in hypoand hyperalphalipoproteinemia. J Lipid Res 2002; 43: 1236-1243.

18. Tamakoshi K, Yatsuya H, Kondo T, Hirano T, Hori Y, Yoshida T, et al. The accuracy of long-term recall of past body weight in Japanese 
adult men. Int J Obes Relat Metab Disord 2003; 27: 247-252.

19. Yatsuya H, Tamakoshi K, Yoshida H, Hori Y, Zhang H, Ishikawa M, et al. Association between weight fluctuation and fasting insulin concentration in Japanese men. Int J Obes Relat Metab Disord 2003; 27: $478-483$.

20. WHO MONICA Project, Tunstall-Pedoe H, Kuulasmaa K, Amouyel $\mathrm{P}$, Arveiler D, Rajakangas AM, Pajak A. Myocardial infarction and coronary deaths in the World Health Organization MONICA Project: Registration procedures, event rates, and case-fatality rates in 38 populations from 21 countries in four continents. Circulation 1994; 90: $583-512$

21. Karkkainen M, Oka T, Olkkonen VM, Metso J, Hattori H, Jauhiainen $\mathrm{M}$, et al. Isolation and partial characterization of the inactive and active forms of human plasma phospholipid transfer protein (PLTP). J Biol Chem 2002; 277: 15413-15418.

22. Oka T, Kujiraoka T, Ito M, Egashira T, Takahashi S, Nanjee MN, et al. Distribution of phospholipid transfer protein in human plasma: Presence of two forms of phospholipid transfer protein, one catalytically active and the other inactive. J Lipid Res 2000; 41: 1651-1657.

23. Yatsuya H, Ohwaki A, Tamakoshi K, Wakai K, Koide K, Otsuka R, et al. Reproducibility and validity of a simple checklist-type questionnaire for food intake and dietary behavior. J Epidemiol 2003; 13: $235-245$.

24. World Health Organization. World health statistics annual 1995. Geneva: WHO, 1996.

25. Schlitt A, Bickel C, Thumma P, Blankenberg S, Rupprecht HJ, Meyer J, et al. High plasma phospholipid transfer protein levels as a risk factor for coronary artery disease. Arterioscler Thromb Vasc Biol 2003; 23: 1857-1862.

26. van Haperen R, van Tol A, van Gent T, Scheek L, Visser P, van der Kamp A, et al. Increased risk of atherosclerosis by elevated plasma levels of phospholipid transfer protein. J Biol Chem 2002; 277: 48938-48943.

27. Kujiraoka T, Nanjee MN, Oka T, Ito M, Nagano M, Cooke CJ, et al. Effects of intravenous apolipoprotein A-I/phosphatidylcholine discs on LCAT, PLTP and CETP in plasma and peripheral lymph in humans. Arterioscler Thromb Vasc Biol 2003; 23: 1653-1659.

28. Jiang XC, Bruce C, Mar J, Lin M, Ji Y, Francone OL, et al. Targeted mutation of plasma phospholipid transfer protein gene markedly reduces high-density lipoprotein levels. J Clin Invest 1999; 103: 907-914.

29. Jiang XC, Qin S, Qiao C, Kawano K, Lin M, Skold A, et al. Apolipoprotein $\mathrm{B}$ secretion and atherosclerosis are decreased in mice with phospholipid-transfer protein deficiency. Nat Med 2001; 7: 847 852.

30. Hattori H, Kujiraoka T, Egashira T, Miyazaki A, Cooper JA, Miller NE. Coronary heart disease is strongly associated with plasma pre-beta-LPA-I concentration: Impact of high density lipoprotein remodeling by cholesteryl ester and phospholipid transfer proteins (abstract). Circulation 2000; 102(Suppl): 1391.

31. Jaari S, van Dijk KW, Olkkonen VM, van der Zee A, Metso J, Havekes L, et al. Dynamic changes in mouse lipoproteins induced by transiently expressed human phospholipid transfer protein (PLTP): Importance of PLTP in prebeta-HDL generation. Comp Biochem Physiol B Biochem Mol Biol 2001; 128: 781-782.

32. Tanabe N, Saito R, Sato T, Hayashi S, Toyoshima H, Seki N, et al. Event rates of acute myocardial infarction and coronary deaths in Niigata and Nagaoka cities in Japan. Circ J 2003; 67: 40-45.

33. Kita Y, Okayama A, Ueshima H, Wada M, Nozaki A, Choudhury SR, et al. Stroke incidence and case fatality in Shiga, Japan 1989-993. Int J Epidemiol 1999; 28: 1059-1065. 